\title{
A NONLINEAR BOUNDARY VALUE PROBLEM ASSOCIATED WITH THE STATIC EQUILIBRIUM OF AN ELASTIC BEAM SUPPORTED BY SLIDING CLAMPS
}

CHAITAN P. GUPTA

Mathematics and Computer Science Division

Argonne National Laboratory

Argonne, IL 60439-4801 U.S.A.

(Received November 2, 1988)

ABSTRACT. The fourth-order boundary value problem $\frac{d^{4} u}{d x^{4}}+f(x) u=e(x), \quad 0<x<\pi$; $u^{\prime}(0)=u^{\prime}(\pi)=u^{\prime \prime \prime}(0)=u^{\prime \prime \prime}(\pi)=0$; where $f(x) \leq 0$ for $0 \leq x \leq \pi$, describe the unstable static equilibrium of an elastic beam which is supported by sliding clamps at both ends. This paper concerns the nonlinear analogue of this boundary value problem, namely, $-\frac{d^{4} u}{d x^{4}}+g(x, u)=e(x), 0<x<\pi, u^{\prime}(0)=u^{\prime}(\pi)=$ $u^{\prime \prime \prime}(0)=u^{\prime \prime \prime}(\pi)=0$, where $g(x, u) u \geq 0$ for a.e. $x$ in $[0, \pi]$ and all $u \in \mathbf{R}$ with $|u|$ sufficiently large. Some resonance and nonresonance conditions on the asymptotic behavior of $u^{-1} g(x, u)$, for $|u|$ sufficiently large, are studied for the existence of solutions of this nonlinear boundary value problem.

KEY WORDS AND PHRASES. elastic beam supported by sliding clamps, asymptotic conditions, resonance, nonresonance, $L^{\infty}$-resonance, Wirtinger's inequalities, coincidence degree theory.

\section{AMS(MOS) SUBJECT CLASSIFICATION CODES. 34B10, 34B15, 73K05}

\section{INTRODUCTION}

The static deformations of an elastic beam supported by sliding clamps at both ends are described by the following fourth-order two-point boundary value problem:

$$
\begin{gathered}
\frac{d^{4} u}{d x^{4}}+f(x) u=e(x), \quad 0<x<\pi, \\
u^{\prime}(0)=u^{\prime}(\pi)=u^{\prime \prime \prime}(0)=u^{\prime \prime \prime}(\pi)=0 .
\end{gathered}
$$

The static equilibrium of the elastic beam described by the boundary value problem (1.1) is said to be unstable if $f(x) \leq 0$, for $0<x<\pi$. This instability is caused by the fact that the term $f(x) u$ may interact with the eigenvalues, $\lambda=n^{4},(n=0,1,2, \ldots)$, for the linear eigenvalue problem

$$
\begin{gathered}
\frac{d^{4} u}{d x^{4}}=\lambda u, \quad 0<x<\pi, \\
u^{\prime}(0)=u^{\prime}(\pi)=u^{\prime \prime \prime}(0)=u^{\prime \prime \prime}(\pi)=0,
\end{gathered}
$$

when $f(x) \leq 0,0<x<\pi$. (1.1):

The purpose of this paper is to study the following nonlinear analogue of the boundary value problem 


$$
\begin{aligned}
& -\frac{d^{4} u}{d x^{4}}+g(x, u)=e(x), \quad 0<x<\pi, \\
& u^{\prime}(0)=u^{\prime}(\pi)=u^{\prime \prime \prime}(0)=u^{\prime \prime \prime}(\pi)=0,
\end{aligned}
$$

where the nonlinear function $g(x, u)$ is such that for some $\rho>0, g(x, u) u \geq 0$ for $x \in[0, \pi], u \in \mathbf{R}$ with $|u| \geq \rho$. More precisely, the purpose of this paper is to give non-resonance and resonance asymptotic conditions at infinity on $g(x, u) u^{-1}$ at the first two eigenvalues $\lambda=0$ and $\lambda=1$ of the linear eigenvalue problem (1.2).

The methods and results of this paper are motivated by the papers of Gupta and Mawhin ([1]) and Mawhin ([2]) (see also [3], [4]) for the second order boundary value problem

$$
\begin{gathered}
\frac{d^{2} u}{d x^{2}}+g(x, u)=e(x), \quad 0<x<2 \pi, \\
u(0)-u(2 \pi)=u^{\prime}(0)-u^{\prime}(2 \pi)=0 .
\end{gathered}
$$

We present in Section 2 some lemmas giving a priori inequalities that are needed to apply degreetheoretic arguments to obtain existence of solutions for the problem (1.3). In Section 3, nonresonance conditions for the existence of solutions of (1.3) are studied, and in Section 4 we study the problem (1.3) when it is at resonance. We sharpen the theorem of Section 4 in Section 5 when (1.3) does not have any $L^{\infty}$ resonance at the second eigenvalue $\lambda=1$ of the linear eigenvalue problem (1.2). Additionally, we present a necessary and sufficient condition that the righthand member $e$ in (1.3) needs to satisfy for the existence of a solution for (1.3) when, among other conditions, $g(x, u)$ is nondecreasing in $u$ for every $x$ in $[0, \pi]$.

In this paper, we use classical spaces $C[0, \pi], C^{k}[0, \pi], L^{k}[0, \pi]$, and $L^{\infty}[0, \pi]$ of continuous, $k$-times continuously differentiable, measurable real-valued functions whose $k$-th power of the absolute value is Lebesgue integrable or measurable functions that are essentially bounded on $[0, \pi]$. In addition, we use the Sobolev-spaces $H^{k}[0, \pi](k=2,3$ or 4$)$ defined by

$$
\begin{aligned}
H^{k}[0, \pi] & =\left\{u:[0, \pi] \rightarrow \mathbf{R} \mid u^{(j)} \text { abs. cont. on }[0, \pi],\right. \\
j & \left.=0,1, \ldots, k-1, \quad u^{(k)} \in L^{2}[0, \pi]\right\}
\end{aligned}
$$

with the inner product defined by

$$
(u, v)_{H^{k}}=\sum_{j=1}^{k} \frac{1}{\pi} \int_{0}^{\pi} u^{(j)}(x) v^{(j)}(x) \mathrm{d} x+\left[\frac{1}{\pi} \int_{0}^{\pi} u(x) \mathrm{d} x\right)\left[\frac{1}{\pi} \int_{0}^{\pi} v(x) \mathrm{d} x\right)
$$

and the corresponding norm by $|\cdot|_{H^{k}}$. We define, for convenience, the norm in $L^{k}[0, \pi]$ by

$$
|u|_{L^{k}}=\left(\frac{1}{\pi} \int_{0}^{\pi}|u(x)|^{k} \mathrm{~d} x\right)^{\frac{1}{k}}
$$

We also use the Sobolev space $W^{4,1}[0, \pi]$ defined by

$$
W^{4,1}[0, \pi]=\left\{u:[0, \pi] \rightarrow \mathbf{R} \mid u^{\prime}, u^{\prime \prime}, u^{\prime \prime \prime}, \text { abs. cont. on }[0, \pi]\right\}
$$

with norm

$$
|u|_{W^{4,1}}=\sum_{j=0}^{4} \int_{0}^{\pi}\left|u^{j}(t)\right| d t
$$




$$
\bar{u}=\frac{1}{\pi} \int_{0}^{\pi} u(x) \mathrm{d} x, \tilde{u}(x)=u(x)-\bar{u}
$$

so that $\int_{0}^{\pi} \tilde{u}(x) \mathrm{d} x=0$. Let $H^{2}[0, \pi]=\left\{u \in H^{2}[0, \pi] \mid \bar{u}=0\right\}$.

LEMMA 1. Let $\Gamma \in L^{1}[0, \pi]$ be such that, for a.e. $x \in[0, \pi]$,

$$
\Gamma(x) \leq 1
$$

with strict inequality holding on a subset of $[0, \pi]$ of positive measure. Then there exists a $\delta=\delta(\Gamma)>0$ such that for all $\tilde{u} \in H^{2}[0, \pi]$ with $\tilde{u}^{\prime}(0)=\tilde{u}^{\prime}(\pi)=0$,

$$
B_{\Gamma}(\tilde{u})=\frac{1}{\pi} \int_{0}^{\pi}\left[\left(\tilde{u}^{\prime \prime}(x)\right)^{2}-\Gamma(x) \tilde{u}^{2}(x)\right] \mathrm{d} x \geq \delta|\tilde{u}|_{H^{2}}^{2}
$$

PROOF. Using (2.2), Wirtinger's inequality [5], and the method of expanding a function $\tilde{u} \in A^{2}[0, \pi]$ with $\tilde{u}^{\prime}(0)=\tilde{u}^{\prime}(\pi)=0$ into a cosine-Fourier series, we see that, for all $\tilde{u} \in A^{2}[0, \pi]$ with $\tilde{u}^{\prime}(0)=\tilde{u}^{\prime}(\pi)=0$,

$$
B_{\Gamma}(\tilde{u}) \geq \frac{1}{\pi} \int_{0}^{\pi}\left[\left(\tilde{u}^{\prime \prime}(x)\right)^{2}-\tilde{u}^{2}(x)\right] \mathrm{d} x \geq 0
$$

Moreover,

$$
B_{\Gamma}(\tilde{u})=0
$$

if and only if

$$
\tilde{u}(x)=A \cos x,
$$

for some $A \in \mathbf{R}$. But then by (2.5),(2.6) we get

$$
\begin{aligned}
0=B_{\Gamma}(\tilde{u}) & =\frac{1}{\pi} \int_{0}^{\pi}[1-\Gamma(x)] \tilde{u}^{2}(x) \mathrm{d} x \\
& =\frac{A^{2}}{\pi} \int_{0}^{\pi}[1-\Gamma(x)] \cos ^{2} x \mathrm{~d} x,
\end{aligned}
$$

so that by our assumption (2.2) on $\Gamma$ we have $A=0$ and hence $\tilde{u}=0$.

Let us next assume that the conclusion of the lemma is false. Then there exists a sequence $\left\{\tilde{u}_{n}\right\}$, $\tilde{u}_{n} \in A^{2}[0, \pi]$ for every $n=1,2,3, \ldots$ such that

$$
\begin{gathered}
B_{\Gamma}\left(\tilde{u}_{n}\right) \rightarrow 0 \text { as } n \rightarrow \infty \\
\left|\tilde{u}_{n}\right|_{H^{2}}=1, \text { for every } n=1,2,3, \ldots .
\end{gathered}
$$

It follows from (2.7) and the compact embedding $H^{2}[0, \pi] \leftrightarrow C^{1}[0, \pi]$ that there exists a $\tilde{u} \in H^{2}[0, \pi]$ such that

$$
\begin{gathered}
\tilde{u}_{n} \rightarrow \tilde{u} \text { weakly in } H^{2}[0, \pi], \\
\tilde{u}_{n} \rightarrow \tilde{u} \text { in } C^{1}[0, \pi] .
\end{gathered}
$$

Now (2.8) implies that $\tilde{u}^{\prime}(0)=\tilde{u}^{\prime}(\pi)=0$ and $|\tilde{u}|_{H^{2}} \leq \liminf _{n \rightarrow \infty}\left|\tilde{u}_{n}\right|_{H^{2}}$. Hence,

$$
0 \leq B_{\Gamma}(\tilde{u}) \leq \liminf _{n \rightarrow \infty} B_{\Gamma}\left(\tilde{u}_{n}\right)=0 \text {. }
$$

It follows from (2.9) and the first part of this proof that $\tilde{u}=0$. Also, (2.7)-(2.9) imply that 


$$
\begin{aligned}
\frac{1}{2 \pi} \int_{0}^{2 \pi}\left[\tilde{u}_{n}^{\prime \prime}(x)\right]^{2} \mathrm{~d} x= & B_{\Gamma}\left(\tilde{u}_{n}\right)+\frac{1}{\pi} \int_{0}^{\pi} \Gamma(x) \tilde{u}_{n}^{2}(x) \mathrm{d} x \\
& \rightarrow \frac{1}{\pi} \int_{0}^{\pi} \Gamma(x) \tilde{u}^{2}(x) \mathrm{d} x=\frac{1}{\pi} \int_{0}^{\pi}\left[\tilde{u}^{\prime \prime}(x)\right]^{2} \mathrm{~d} x,
\end{aligned}
$$

so that $\tilde{u}_{n} \rightarrow \tilde{u}$ in $H^{2}[0, \pi]$ and $|\tilde{u}|_{/ I^{2}}=1$. We have thus arrived at a contradiction.

LEMMA 2. Let $\Gamma=\Gamma_{0}+\Gamma_{1}+\Gamma_{\infty}$ where $\Gamma_{\infty} \in L^{\infty}[0, \pi], \Gamma_{1} \in L^{1}[0, \pi]$, and $\Gamma_{0} \in L^{1}[0, \pi]$ be such that $\Gamma_{0}(x) \leq 1$ for a.e. $x \in[0, \pi]$ with strict inequality holding on a subset of $[0, \pi]$ of positive measure. Let $\delta\left(\Gamma_{0}\right)>0$ be as given by Lemma 1. Then for every $\tilde{u} \in H^{2}[0, \pi]$ with $\tilde{u}^{\prime}(0)=\tilde{u}^{\prime}(\pi)=0$,

$$
B_{\Gamma}(\tilde{u}) \geq\left[\delta\left(\Gamma_{0}\right)-\frac{\pi^{2}}{3}\left|\Gamma_{1}\right|_{L^{1}}-\left|\Gamma_{\infty}\right|_{L^{-}}\right]|\tilde{u}|_{H^{2}}^{2}
$$

PROOF. We have

$$
\begin{aligned}
B_{\Gamma}(\tilde{u})= & \frac{1}{\pi} \int_{0}^{\pi}\left[\left(\tilde{u}^{\prime \prime}(x)\right)^{2}-\Gamma_{0}(x) \tilde{u}^{2}(x)\right] \mathrm{d} x \\
& -\frac{1}{\pi} \int_{0}^{\pi} \Gamma_{1}(x) \tilde{u}^{2}(x) \mathrm{d} x-\frac{1}{\pi} \int_{0}^{\pi} \Gamma_{\infty}(x) \tilde{u}^{2}(x) \mathrm{d} x .
\end{aligned}
$$

Using, now, the fact that $H^{2}[0, \pi] \subset C^{1}[0, \pi]$ and the inequalities (see [9])

$$
|\tilde{u}|_{L^{2}} \leq\left|\tilde{u}^{\prime}\right|_{L^{2}} \leq|\tilde{u}|_{H^{2}}, \quad|\tilde{u}|_{L^{-}} \leq \frac{\pi}{\sqrt{3}}\left|\tilde{u}^{\prime}\right|_{L^{2}} \leq \frac{\pi}{\sqrt{3}}|\tilde{u}|_{H^{2}}
$$

for $\tilde{u} \in H^{2}[0, \pi]$ with $\tilde{u}^{\prime}(0)=\tilde{u}^{\prime}(\pi)=0$, as well as Lemma 1 , we get that

$$
\begin{aligned}
B_{\Gamma}(\tilde{u}) & \geq \delta\left(\Gamma_{0}\right)|\tilde{u}|_{H^{2}}^{2}-\left|\Gamma_{1}\right|_{L^{1}} \cdot|\tilde{u}|_{L^{-}}^{2}-\left|\Gamma_{\infty}\right|_{L^{-}} \cdot|\tilde{u}|_{L^{2}}^{2} \\
& \geq\left[\delta\left(\Gamma_{0}\right)-\frac{\pi^{2}}{3}\left|\Gamma_{1}\right|_{L^{1}}-\left|\Gamma_{\infty}\right|_{L^{-}}\right]|\tilde{u}|_{H^{2}}^{2} .
\end{aligned}
$$

Remark 1. The best value for $\delta(0)$ is clearly $\frac{1}{2}$, so that $B_{\Gamma_{1}}(\tilde{u}) \geq\left(\frac{1}{2}-\pi^{2}\left|\Gamma_{1}\right|_{L^{1}}\right)|\tilde{u}|_{H^{2}}^{2}$ for all $\tilde{u} \in H^{2}[0, \pi]$ with $\tilde{u}^{\prime}(0)=\tilde{u}^{\prime}(\pi)=0$.

LEMMA 3. Let $\gamma \in L^{1}[0, \pi], \Gamma=\Gamma_{0}+\Gamma_{1}+\Gamma_{\infty}$ be as in Lemma 2 and $\delta\left(\Gamma_{0}\right)>0$ be given by Lemma 1 . Then for all measurable functions $p(x)$ on $[0, \pi]$ such that $\bar{\gamma} \leq \bar{p}, p(x) \leq \Gamma(x)$ for a.e. $x \in[0, \pi]$ and all $u \in W^{4,1}[0, \pi]$ with $u^{\prime}(0)=u^{\prime}(\pi)=u^{\prime \prime \prime}(0)=u^{\prime \prime \prime}(\pi)=0$, we have

$$
\begin{aligned}
& \frac{1}{\pi} \int_{0}^{\pi}[\bar{u}-\tilde{u}(x)]\left[-\tilde{u}^{(i v)}(x)+p(x) u(x)\right] \mathrm{d} x \\
& \quad \geq \overline{\gamma u}^{2}+\left[\delta\left(\Gamma_{0}\right)-\frac{\pi^{2}}{3}\left|\Gamma_{1}\right|_{L^{1}}-\left|\Gamma_{\infty}\right|_{L^{-}}\right]|\tilde{u}|_{H^{2}}^{2} .
\end{aligned}
$$

PROOF. For $u \in W^{4,1}[0, \pi]$ with $u^{\prime}(0)=u^{\prime}(\pi)=u^{\prime \prime \prime}(0)=u^{\prime \prime \prime}(\pi)=0$, we have (on integrating by parts and using Lemma 2) that

$$
\begin{aligned}
\frac{1}{\pi} \int_{0}^{\pi}[\tilde{u} & -\tilde{u}(x)]\left[-u^{(i v)}(x)+p(x) u(x)\right] \mathrm{d} x \\
& \geq \bar{p} \cdot \bar{u}^{2}+\frac{1}{\pi} \int_{0}^{\pi}\left[\left(\tilde{u}^{\prime \prime}(x)\right)^{2}-p(x) \tilde{u}^{2}(x)\right] \mathrm{d} x
\end{aligned}
$$




$$
\geq \bar{\gamma} \cdot \bar{u}^{2}+\left[\delta\left(\Gamma_{0}\right)-\frac{\pi^{2}}{3}\left|\Gamma_{1}\right|_{L^{1}}-\left|\Gamma_{\infty}\right|_{L^{-}}\right]|\tilde{u}|_{H^{2}}^{2}
$$

\section{NONRESONANCE CONDITIONS FOR THE EXISTENCE OF SOLUTIONS}

Let $g:[0, \pi] \times \mathbf{R} \rightarrow \mathbf{R}$ be a function satisfying Caratheodory conditions, namely,

(i) for each $u \in \mathbf{R}$, the function $x \in[0, \pi] \rightarrow g(x, u) \in \mathbf{R}$ is measurable on $[0, \pi]$;

(ii) for a.e. $x \in[0, \pi]$, the function $u \in \mathbf{R} \rightarrow g(x, u) \in \mathbf{R}$ is continuous on $\mathbf{R}$; and

(iii) for each $r>0$, there exists a function $\alpha_{r}(x) \in L^{1}[0, \pi]$ such that $|g(x, u)| \leq \alpha_{r}(x)$ for a.e. $x \in[0, \pi]$ and all $u \in \mathbf{R}$ with $|u| \leq r$.

THEOREM 1. Let $\gamma \in L^{1}[0, \pi]$ with $\bar{\gamma}>0$ be given. Also let $\Gamma=\Gamma_{0}+\Gamma_{1}+\Gamma_{\infty}$ with $\Gamma_{1} \in L^{1}[0, \pi]$, $\Gamma_{\infty} \in L^{\infty}[0, \pi], \Gamma_{0}$ measurable on $[0, \pi], \Gamma_{0}(x) \leq 1$ with strict inequality holding on a subset of $[0, \pi]$ of positive measure, and $\frac{\pi^{2}}{3}\left|\Gamma_{1}\right|_{L^{1}}+\left|\Gamma_{\infty}\right|_{L^{-}}<\delta\left(\Gamma_{0}\right)$, where $\delta\left(\Gamma_{0}\right)>0$, is given by Lemma 1. Assume that the inequalities

$$
\gamma(x) \leq \liminf _{|u| \rightarrow \infty} u^{-1} g(x, u) \leq \limsup _{|u| \rightarrow \infty} u^{-1} g(x, u) \leq \Gamma(x),
$$

- hold uniformly for a.e. $x \in[0, \pi]$.

Then, for every given $e(x) \in L^{1}[0, \pi]$ the boundary value problem (1.3) has at least one solution.

PROOF. Let $\eta=\frac{1}{2} \min \left\{\bar{\gamma}, \delta\left(\Gamma_{0}\right)-\frac{\pi^{2}}{3}\left|\Gamma_{1}\right|_{L^{1}}-\left|\Gamma_{\infty}\right|_{L^{-}}\right\}>0$. Then, by (3.1) we can find an $r>0$ such that for a.e. $x \in[0, \pi]$ and every $u \in \mathbf{R}$ with $|u| \geq r$ we have

$$
\gamma(x)-\eta \leq g(x, u) u^{-1} \leq \Gamma(x)+\eta .
$$

Next, define $\tilde{\boldsymbol{\gamma}}:[0, \pi] \times \mathbf{R} \rightarrow \mathbf{R}$ by

$$
\tilde{\gamma}(x, u)=\left\{\begin{array}{cc}
u^{-1} g(x, u) & \text { if }|u| \geq r \\
r^{-1} g(x, r) & \text { if } 0<u<r \\
-r^{-1} g(x,-r) & \text { if }-r<u<0 \\
\Gamma(x) & \text { if } u=0 .
\end{array}\right.
$$

Note that $\tilde{\gamma}(x, u) u$ satisfies Caratheodory's conditions and, from (3.2),

$$
\gamma(x)-\eta \leq \tilde{\gamma}(x, u) \leq \Gamma(x)+\eta
$$

for a.e. $x \in[0, \pi]$ and all $u \in \mathbf{R}$. Now, define $h:[0, \pi] \times \mathbf{R} \rightarrow \mathbf{R}$ by

$$
h(x, u)=g(x, u)-\tilde{\gamma}(x, u) u,
$$

for $x \in[0, \pi], u \in \mathbf{R}$. We then see that

$$
\begin{aligned}
|h(x, u)| & \leq \sup _{|u| \leq r}|g(x, u)-\tilde{\gamma}(x, u) u| \\
& \leq \alpha(x),
\end{aligned}
$$

for $x \in[0, \pi], u \in \mathbf{R}$, where $\alpha(x) \in L^{1}[0, \pi]$ depends on $\gamma, \Gamma$, and $\alpha_{r}$.

The equation in (1.3) is equivalent to the equation

$$
-\frac{d^{4} u}{d x^{4}}+\tilde{\gamma}(x, u(x)) u(x)+h(x, u(x))=e(x),
$$

to which we apply coincidence degree theory $[6,7]$ in a manner similar to the method used in Theorem 1 of [3]. Let $X=C[0, \pi], Z=L^{1}[0, \pi], \operatorname{dom} L=\left\{u \in W^{4,1}[0, \pi] \mid u^{\prime}(0)=u^{\prime}(\pi)=u^{\prime \prime \prime}(0)=u^{\prime \prime \prime}(\pi)=0\right\}$. 


$$
\begin{aligned}
& L: \operatorname{dom} L \subset X \rightarrow Z, u \rightarrow-\frac{d^{4} u}{d x^{4}} \\
& G: X \rightarrow Z, u \rightarrow \tilde{\gamma}(\cdot, u(\cdot)) u(\cdot) \\
& H: X \rightarrow Z, u \rightarrow h(\cdot, u(\cdot))-e(\cdot) \\
& A: X \rightarrow Z, u \rightarrow \tilde{\gamma}(\cdot, 0) u(\cdot)=\Gamma(\cdot) u(\cdot) .
\end{aligned}
$$

It is easy to check that $G, H, A$ are well defined and $L$-compact on bounded subsets of $X$ and that $L$ is a linear Fredholm mapping of index zero. We consider the homotopy $\Phi$ : $\operatorname{dom} L \times[0, \pi] \rightarrow Z$ defined by

$$
\Phi(u, \lambda) \equiv L u+(1-\lambda) A u+\lambda G u+\lambda H u,
$$

for $u \in \operatorname{dom} L, \lambda \in[0,1]$. Now, in order to apply Theorem IV.5 of [7] (see also [8],[9]), it suffices to show that the set of possible solutions of the family of equations

$$
\begin{gathered}
-\frac{d^{4} u}{d x^{4}}+[(1-\lambda) \Gamma(x)+\lambda \tilde{\gamma}(x, u(x))] u(x)+\lambda h(x, u(x))-\lambda e(x)=0, \lambda \in(0,1) \\
u^{\prime}(0)=u^{\prime}(\pi)=u^{\prime \prime \prime}(0)=u^{\prime \prime \prime}(\pi)=0,
\end{gathered}
$$

is, a priori bounded in $C[0, \pi]$ independently of $\lambda \in(0,1)$. If $u$ is a solution of (3.6), then multiplying (3.6) by $\bar{u}-\tilde{u}$, integrating over $[0, \pi]$, and using (3.3),(3.5) together with Lemma 3 with $\Gamma_{\infty}$ replaced by $\Gamma_{\infty}+\eta$ and $\gamma$ replaced by $\gamma-\eta$, we get

$$
\begin{aligned}
0 & =\frac{1}{\pi} \int_{0}^{\pi}(\bar{u}-\tilde{u}(x))\left\{-\frac{d^{4} u}{d x^{4}}+[(1-\lambda) \Gamma(x)+\tilde{\gamma}(x, u(x))] u(x)+\lambda h(x, u(x))-\lambda l(x)\right\} \mathrm{d} x \\
& \geq(\bar{\gamma}-\eta) \bar{u}^{2}+\left[\delta\left(\Gamma_{0}\right)-\frac{\pi^{2}}{3}\left|\Gamma_{1}\right|_{L^{1}}-\left|\Gamma_{\infty}\right|_{L^{-}}-\eta\right]|\tilde{u}|_{H^{2}}^{2}-\left(|\alpha|_{L^{1}}+|e|_{L^{1}}\right)|\bar{u}-\tilde{u}|_{L^{-}} \\
& \geq \frac{\bar{\gamma}}{2} \bar{u}^{2}+\frac{1}{2}\left[\delta\left(\Gamma_{0}\right)-\frac{\pi^{2}}{3}\left|\Gamma_{1}\right|_{L^{1}}-\left|\Gamma_{\infty}\right|_{L^{-}}\right]|\tilde{u}|_{H^{2}}^{2}-\beta|u|_{H^{2}}
\end{aligned}
$$

for some constant $\beta>0$, independent of $\lambda \in[0,1]$. It then follows that $|u|_{H^{2}} \leq \beta / \eta$, which implies that

$$
|u|_{C[0, \pi]} \leq C,
$$

where $C$ is a constant independent of $\lambda \in[0,1]$, in view of the compact imbedding of $H^{2}[0, \pi] \subset C[0,1]$.

COROLLARY 1. Let $\gamma$ and $\Gamma=\Gamma_{0}+\Gamma_{1}+\Gamma_{\infty}$ be as in Theorem 1 above. Then, for every given $e(x) \in L^{1}[0, \pi]$ the boundary value problem

$$
\begin{aligned}
& -\frac{d^{4} u}{d x^{4}}+\Gamma(x) u=e(x), \quad 0<x<\pi \\
& u^{\prime}(0)=u^{\prime}(\pi)=u^{\prime \prime \prime}(0)=u^{\prime \prime \prime}(\pi)=0,
\end{aligned}
$$

has exactly one solution.

PROOF. The existence of a solution for (3.7) is immediate from Theorem 1 since $g(x, u)=\Gamma(x) u$ obviously satisfies all the conditions of Theorem 1 .

Let $u_{1}(x), u_{2}(x)$ be two solutions of (3.5). Setting $v(x)=u_{1}(x)-u_{2}(x)$, we get that 


$$
\begin{gathered}
-\frac{d^{4} v}{d x^{4}}+\Gamma(x) v(x)=0, \quad 0<x<\pi, \\
v^{\prime}(0)=v^{\prime}(\pi)=v^{\prime \prime \prime}(0)=v^{\prime \prime \prime}(\pi)=0 .
\end{gathered}
$$

Multiplying the equation in (3.8) by $\bar{v}-\tilde{v}$, integrating by parts on $[0, \pi]$, and using Lemma 3 , we get that

$$
\begin{aligned}
0 & =\int_{0}^{\pi}[\bar{v}-\bar{v}(x)]\left\{-\frac{d^{4} v}{d x^{4}}+\Gamma(x) v(x)\right\} d x \\
& \geq \bar{\gamma}^{2}+\left[\delta\left(\Gamma_{0}\right)-\frac{\pi^{2}}{3}\left|\Gamma_{1}\right|_{L^{1}}-\left|\Gamma_{\infty}\right|_{L^{-}}\right]|\tilde{v}|_{H^{2}}^{2} \geq 0 .
\end{aligned}
$$

Thus $\bar{v}=0$ and $|\tilde{v}|_{H^{2}}=0$. Since, now,

$$
|\tilde{v}|_{L^{-\infty}} \leq \frac{\pi}{\sqrt{3}}\left|\tilde{v}^{\prime}\right|_{L^{2}} \leq \frac{\pi}{\sqrt{3}}|\tilde{v}|_{H^{2}}=0,
$$

we get $\tilde{v}=0$ and hence $v=\bar{v}+\tilde{v}=0$.

\section{RESONANCE CONDITIONS FOR THE EXISTENCE OF SOLUTIONS}

Let $g:[0, \pi] \times \mathbf{R} \rightarrow \mathbf{R}$ be a function satisfying Caratheodory's conditions.

THEOREM 2. Let $\Gamma \in L^{1}[0,2 \pi]$ be such that

$$
\limsup _{|u| \rightarrow \infty} \frac{g(x, u)}{u} \leq \Gamma(x)
$$

uniformly for a.e. $x \in[0, \pi]$ and $\Gamma=\Gamma_{0}+\Gamma_{1}+\Gamma_{\infty}$ where $\Gamma_{\infty} \in L^{\infty}[0, \pi], \Gamma_{1} \in L^{1}[0, \pi]$, and $\Gamma_{0} \in L^{1}[0, \pi]$ are such that $\Gamma_{0}(x) \leq 1$ for a.e. $x \in[0, \pi]$ with strict inequality holding on a subset of $[0, \pi]$ of positive measure and $\left|\Gamma_{\infty}\right|_{L^{-}}+\frac{\pi^{2}}{3}\left|\Gamma_{1}\right|_{L^{1}}<\delta\left(\Gamma_{0}\right)$, where $\delta\left(\Gamma_{0}\right)>0$ is given by Lemma 3. Suppose, further, that there exist real numbers $a, A, r$, and $R$ with $a \leq A$ and $r<0<R$ such that

$$
g(x, u) \geq A
$$

for a.e. $x \in[0, \pi]$ and all $u \geq R$, and

$$
g(x, u) \leq a
$$

for a.e. $x \in[0, \pi]$ and all $u \leq r$.

Then, for every given $e(x) \in L^{1}[0, \pi]$ with $a \leq \bar{e} \leq A$, the boundary value problem

$$
\begin{gathered}
-\frac{d^{4} u}{d x^{4}}+g(x, u(x))=e(x), \quad 0<<\pi, \\
u^{\prime}(0)=u^{\prime}(\pi)=u^{\prime \prime \prime}(0)=u^{\prime \prime \prime}(\pi)=0
\end{gathered}
$$

has at least one solution.

PROOF. Define $g_{1}:[0, \pi] \times \mathbf{R} \rightarrow \mathbf{R}$ by $g_{1}(x, u)=g(x, u)-\frac{1}{2}(a+A)$ and $e_{1} \in L^{1}[0, \pi]$ by $e_{1}(x)=e(x)-\frac{1}{2}(a+A)$, so that for a.e. $x \in[0, \pi]$ we have, by using (4.2),(4.3), and the assumption $a \leq \bar{e} \leq A$, that

$$
\begin{aligned}
& g_{1}(x, u) \geq \frac{1}{2}(A-a) \geq 0 \text { if } u \geq R, \\
& g_{1}(x, u) \leq \frac{1}{2}(a-A) \leq 0 \text { if } u \leq r,
\end{aligned}
$$


and

$$
\frac{1}{2}(a-A) \leq \bar{e}_{1} \leq \frac{1}{2}(A-a) .
$$

The equation in (4.4) is clearly equivalent to the equation

$$
-\frac{d^{4} u}{d x^{4}}+g_{1}(x, u(x))=e_{1}(x), \quad 0<x<\pi .
$$

Moreover, we have

$$
\limsup _{|u| \rightarrow \infty} u^{-1} g_{1}(x, u) \leq \Gamma(x)
$$

uniformly for a.e. $x \in[0, \pi]$ and for $|u| \geq \max (R,-r)$, a.e. $x \in[0, \pi], g_{1}(x, u) u \geq 0$. Hence, $\Gamma(x) \geq 0$ for a.e. $x \in[0, \pi]$.

Now let $\eta=\frac{1}{2}\left[\delta\left(\Gamma_{0}\right)-\frac{\pi^{2}}{3}\left|\Gamma_{1}\right|_{L^{1}}-\left|\Gamma_{\infty}\right|_{L^{-}}\right]>0$. Then, there exists an $r_{1}>0$ such that for a.e. $x \in[0, \pi]$ and for all $u \in \mathbf{R},|u| \geq r_{1}$, we have

$$
0 \leq u^{-1} g_{1}(x, u) \leq \Gamma(x)+\eta .
$$

Proceeding as in the proof of Theorem 1, we write the equation (4.8) in the equivalent form

$$
-\frac{d^{4} u}{d x^{4}}+\tilde{\gamma}(x, u(x)) u(x)+h(x, u(x))=e_{1}(x),
$$

where $0 \leq \tilde{\gamma}(x, u) \leq \Gamma(x)+\eta,|h(x, u)| \leq \alpha(x)$, for a.e. $x \in[0, \pi]$, all $u \in \mathbf{R}$ and some $\alpha \in L^{1}[0, \pi]$. Once again degree arguments will ensure the existence of a solution for (4.4) if the set of all possible solutions of the family of equations

$$
\begin{aligned}
& -\frac{d^{4} u}{d x^{4}}+[(1-\lambda)(\Gamma(x)+\eta)+\lambda \tilde{\gamma}(x, u(x))] u(x) \\
& +\lambda h(x, u(x))=\lambda e_{1}(x), \quad \lambda \in(0,1) \\
& u^{\prime}(0)=u^{\prime}(\pi)=u^{\prime \prime \prime}(0)=u^{\prime \prime \prime}(\pi)=0,
\end{aligned}
$$

is, a priori, bounded in $C[0, \pi]$ independently of $\lambda \in(0,1)$. If, now, $u(x)$ is a possible solution of $(4.11)$ for some $\lambda \in(0,1)$, then integrating the equation in $(4.11)$ over $[0, \pi]$ after multiplying it by $\bar{u}-\tilde{u}$, we get (using Lemma 3 with $\gamma=0$, and $\Gamma_{\infty}$ replaced by $\Gamma_{\infty}+\eta$ )

$$
\begin{aligned}
0 & =\frac{1}{\pi} \int_{0}^{\pi}[\bar{u}-\tilde{u}(x)]\left\{-\frac{d^{4} u}{d x^{4}}+[(1-\lambda)(\Gamma(x)+\eta)+\tilde{\gamma}(x, u(x))] u(x)+\lambda h(x, u(x))-\lambda e_{1}(x)\right\} \mathrm{d} x \\
& \geq\left[\delta\left(\Gamma_{0}\right)-\frac{\pi^{2}}{3}\left|\Gamma_{1}\right|_{L^{1}}-\left|\Gamma_{\infty}\right|_{L^{-}}-\eta\right]|\tilde{u}|_{H^{2}}^{2}-\left(|\alpha|_{L^{1}}+\left|e_{1}\right|_{L^{1}}\right)|\bar{u}-\tilde{u}|_{L^{-}} \\
& \geq \eta|\tilde{u}|_{H^{2}}^{2}-\beta\left(|\bar{u}|+|\tilde{u}|_{H^{2}}\right)
\end{aligned}
$$

for some constant $\beta>0$, irdependent of $\lambda \in(0,1)$. Hence

$$
|\tilde{u}|_{H^{2}}^{2} \leq(\beta / \eta)\left(|\bar{u}|+|\tilde{u}|_{H^{2}}\right) \text {. }
$$

Next, integrating the equation in (4.11) over $[0, \pi]$, we get 


$$
\frac{1}{\pi}(1-\lambda) \int_{0}^{\pi}(\Gamma(x)+\eta) u(x) \mathrm{d} x+\frac{1}{\pi} \lambda \int_{0}^{\pi}\left[g_{1}(x, u(x))-e_{1}(x)\right] \mathrm{d} x=0 .
$$

If $u(x) \geq R$ for all $x \in[0, \pi]$, then (4.5) and (4.7) imply that $(1-\lambda)(\bar{\Gamma}+\eta) R \leq 0$, contradicting $\bar{\Gamma}+\eta \geq \eta>0$. Similarly, $u(x) \leq r$ for all $x \in[0, \pi]$ leads to a contradiction. Thus, there must exist a $\tau \in[0, \pi]$ such that

$$
r<u(\tau)<R
$$

It is easy to see from $u(x)=u(\tau)+\int_{\tau}^{x} u^{\prime}(s) d s$ that

$$
|\bar{u}| \leq \max (R,-r)+|\tilde{u}|_{H^{2}} .
$$

The inequalities (4.12) and (4.14) now imply that

$$
|\tilde{u}|_{H^{2}}^{2} \leq(2 \beta / \eta)|\tilde{u}|_{H^{2}}+(\beta / \eta) \max (R,-r),
$$

so there exists a constant $\rho$, independent of $\lambda \in(0,1)$ such that

$$
|\tilde{u}|_{H^{2}} \leq \rho \text {. }
$$

Finally, (4.14) and (4.15) imply that there is a constant $C$ independent of $\lambda \in(0,1)$ such that

$$
|u|_{H^{2}} \leq C,
$$

which implies that $|u|_{C[0, \pi]} \leq C_{1}$, for some constant $C_{1}$, independent of $\lambda \in(0,1)$.

Remark 2. We say that the boundary value problem (4.4) has "no $L^{\infty}$-resonance" at the second eigenvalue $\lambda=1$, of the linear eigenvalue problem $\frac{d^{4} u}{d x^{4}}=\lambda u, u^{\prime}(0)=u^{\prime}(\pi)=u^{\prime \prime \prime}(0)=u^{\prime \prime \prime}(\pi)=0$, if $\Gamma_{0}=\Gamma_{\infty}=0$ in Theorem 2. In the case of no $L^{\infty}$-resonance, Theorem 2 implies the existence of a solution for the boundary value problem (4.4) if $\left|\Gamma_{1}\right|_{L^{1}}<\frac{3}{2 \pi^{2}}$. We develop this result further in Section 5.

\section{RESONANCE CONDITION WHEN NO $L^{\infty}$-RESONANCE EXISTS}

We need the following lemma for a sharper resonance condition which gives the existence of a solution for the boundary value problem (4.4) when there is no $L^{\infty}$-resonance.

LEMMA 4. Let $e \in L^{1}[0, \pi], \Gamma \in L^{1}[0, \pi]$ with $\bar{\Gamma} \geq 0$. Then every possible solution $u(x)$ of the linear boundary value problem

$$
\begin{gathered}
-\frac{d^{4} u}{d x^{4}}+p(x) u(x)=e(x), \quad 0<x<\pi, \\
u^{\prime}(0)=u^{\prime}(\pi)=u^{\prime \prime \prime}(0)=u^{\prime \prime \prime}(\pi)=0
\end{gathered}
$$

with $p \in L^{1}[0, \pi]$ such that

$$
\bar{p} \leq \bar{\Gamma}, \quad 0 \leq p(x)
$$

for a.e. $x \in[0, \pi]$, satisfies the inequality

$$
\left(1-\frac{\pi^{2}}{4} \bar{\Gamma}\right)\left|\frac{d^{4} u}{d x^{4}}\right|_{L^{1}}^{2} \leq 2|e|_{L^{1}}\left|\frac{d^{4} u}{d x^{4}}\right|_{L^{1}}+\bar{\Gamma}|e|_{L^{1}} \cdot|u|_{L^{-}}+3|e|_{L^{1}}^{2}
$$

PROOF. Let $p \in L^{1}[0, \pi]$ be as in the lemma, and let $u(x)$ be a solution of (5.1). Then, on multiplying the equation in (5.1) by $\frac{1}{\pi} u(x)$ and integrating over $[0, \pi]$, we get 


$$
-\frac{1}{\pi} \int_{0}^{\pi}\left(u^{\prime \prime}(x)\right)^{2} \mathrm{~d} x+\frac{1}{\pi} \int_{0}^{\pi} p(x) u^{2}(x) \mathrm{d} x=\frac{1}{\pi} \int_{0}^{\pi} e(x) u(x) \mathrm{d} x .
$$

Since $\bar{p} \leq \bar{\Gamma}$, we have, by using Schwarz's inequality,

$$
\begin{aligned}
{\left[\frac{1}{\pi} \int_{0}^{\pi}|p(x) u(x)| \mathrm{d} x\right]^{2} } & \leq\left(\frac{1}{\pi} \int_{0}^{\pi} p(x) \mathrm{d} x\right)\left[\frac{1}{\pi} \int_{0}^{\pi} p(x) u^{2}(x) \mathrm{d} x\right) \\
& \leq \bar{\Gamma}\left(\frac{1}{\pi} \int_{0}^{\pi} p(x) u^{2}(x) \mathrm{d} x\right),
\end{aligned}
$$

and hence, using the equation in (5.1),

$$
\left.\left|\frac{1}{\pi} \int_{0}^{\pi}\right| e(x)+\frac{d^{4} u}{d x^{4}} \mid \mathrm{d} x\right]^{2} \leq \bar{\Gamma}\left[\frac{1}{\pi} \int_{0}^{\pi} p(x) u^{2}(x) \mathrm{d} x\right) .
$$

Since $u^{\prime \prime \prime}(0)=u^{\prime \prime \prime}(\pi)=0$, we have

$$
u^{\prime \prime \prime}(x)=\int_{0}^{x} \frac{d^{4} u}{d x^{4}}(s) d s=-\int_{x}^{\pi} \frac{d^{4} u}{d x^{4}}(s) d s,
$$

so that

$$
2\left|u^{\prime \prime \prime}(x)\right| \leq \int_{0}^{\pi}\left|\frac{d^{4} u}{d x^{4}}\right| \mathrm{d} x
$$

Hence,

$$
\frac{1}{\pi} \int_{0}^{\pi}\left|u^{\prime \prime \prime}(x)\right|^{2} \mathrm{~d} x \leq \frac{\pi^{2}}{4}\left[\frac{1}{\pi} \int_{0}^{\pi}\left|\frac{d^{4} u}{d x^{4}}\right| \mathrm{d} x\right]^{2} .
$$

Now, we get from (5.4), (5.6), and (5.7) that

$$
\begin{aligned}
& \frac{1}{\bar{\Gamma}}\left|\frac{1}{\pi} \int_{0}^{\pi}\right| e(x)+\frac{d^{4} u}{d x^{4}}|\mathrm{~d} x|^{2}+\frac{1}{\pi} \int_{0}^{\pi}\left(u^{\prime \prime \prime}(x)\right)^{2} \mathrm{~d} x \\
& \leq \frac{1}{\pi} \int_{0}^{\pi}\left(u^{\prime \prime}(x)\right)^{2} \mathrm{~d} x+\frac{1}{\pi} \int_{0}^{\pi} e(x) u(x) \mathrm{d} x+\frac{\pi^{2}}{4}\left|\frac{1}{\pi} \int_{0}^{\pi}\right| \frac{d^{4} u}{d x^{4}}|\mathrm{~d} x|^{2} .
\end{aligned}
$$

So,

$$
\begin{aligned}
& -\frac{\pi^{2}}{4}\left|\frac{d^{4} u}{d x^{4}}\right|_{L^{1}}^{2}+\frac{1}{\bar{\Gamma}}\left|e(x)+\frac{d^{4} u}{d x^{4}}\right|_{L^{1}}^{2} \leq\left|u^{\prime \prime}\right|_{L^{2}}^{2}-\left|u^{\prime \prime \prime}\right|_{L^{2}}^{2}+|e|_{L^{1}} \cdot|u|_{L^{-}} \\
& \leq|e|_{L^{1}} \cdot|u|_{L^{-}},
\end{aligned}
$$

which then gives that 
Finally,

$$
-\frac{\pi^{2}}{4} \bar{\Gamma}\left|\frac{d^{4} u}{d x^{4}}\right|_{L^{1}}^{2}+\left|e(x)+\frac{d^{4} u}{d x^{4}}\right|_{L^{1}}^{2} \leq \bar{\Gamma}|e|_{L^{1}} \cdot|u|_{L^{-}}
$$

$$
\begin{aligned}
{\left[1-\frac{\pi^{2}}{4} \bar{\Gamma}\right)\left|\frac{d^{4} u}{d x^{4}}\right|_{L^{1}}^{2} } & =\left|\frac{d^{4} u}{d x^{4}}+e(x)-e(x)\right|_{L^{1}}^{2}-\frac{\pi^{2}}{4} \bar{\Gamma}\left|\frac{d^{4} u}{d x^{4}}\right|_{L^{1}}^{2} \\
& \leq\left|\frac{d^{4} u}{d x^{4}}+e(x)\right|_{L^{1}}^{2}+2|e|_{L^{1}} \cdot\left|\frac{d^{4} u}{d x^{4}}+e(x)\right|_{L^{1}}+|e|_{L^{1}}^{2}-\frac{\pi^{2}}{4} \bar{\Gamma}\left|\frac{d^{4} u}{d x^{4}}\right|_{L^{1}}^{2} \\
& \leq 2|e|_{L^{1}} \cdot\left|\frac{d^{4} u}{d x^{4}}\right|_{L^{1}}+\bar{\Gamma}|e|_{L^{1}} \cdot|u|_{L^{-*}}+3|e|_{L^{1}}^{2} .
\end{aligned}
$$

THEOREM 3. Let $g:[0, \pi] \times \mathbf{R} \rightarrow \mathbf{R}$ be a function satisfying Casatheodory's conditions. Assume that there exists $a \Gamma \in L^{1}[0, \pi]$ such that

$$
\lim _{|u| \rightarrow \infty} \sup ^{-1} g(x, u) \leq \Gamma(x)
$$

uniformly for a.e. $x \in[0, \pi]$ and that $\bar{\Gamma}<\frac{4}{\pi^{2}}$. Suppose further that there exist real numbers $a, A, r, R$ with $a \leq A$ and $r<0<R$ such that for a.e. $x \in[0, \pi], g(x, u) \geq A$ when $u \geq R$ and $g(x, u) \leq a$ when $u \leq r$. Then the boundary value problem

$$
\begin{gathered}
-\frac{d^{4} u}{d x^{4}}+g(x, u(x))=e(x), \quad 0<x<\pi, \\
u^{\prime}(0)=u^{\prime}(\pi)=u^{\prime \prime \prime}(0)=u^{\prime \prime \prime}(\pi)=0,
\end{gathered}
$$

has at least one solution for each given $e \in L^{1}[0, \pi]$ with $a \leq \bar{e} \leq A$.

PROOF. We first define $g_{1}$ and $e_{1}$ as in the proof of Theorem 2 so that the equation in (5.8) can be written as

$$
-\frac{d^{4} u}{d x^{4}}+g_{1}(x, u(x))=e_{1}(x),
$$

with $g_{1}(x, u) \geq 0$ where $u \geq R$ and $g_{1}(x, u) \leq 0$ when $u \leq r$ for a.e. $x \in[0, \pi]$ and $\lim \sup u^{-1} g_{1}(x, u) \leq \Gamma(x)$ uniformly for a.e. $x \in[0, \pi]$. Consequently, for a.e. $x \in[0, \pi], \Gamma(x) \geq 0$. Let $\eta=\frac{1}{2}\left[\frac{4}{\pi^{2}}-\bar{\Gamma}\right]>0$, so that $\bar{\Gamma}+\eta<\frac{4}{\pi^{2}}$ and let $r_{1}>0$ be such that

$$
0 \leq u^{-1} g_{1}(x, u) \leq \Gamma(x)+\eta
$$

for a.e. $x \in[0, \pi],|u| \geq r_{1}$. proceeding as in the proof of Theorem 1, we can write (5.9) in the form

$$
-\frac{d^{4} u}{d x^{4}}+\tilde{\gamma}(x, u(x)) u(x)+h(x, u(x))=e_{1}(x),
$$

where $0 \leq \tilde{\gamma}(x, u) \leq \Gamma(x)+\eta,|h(x, u)| \leq \alpha(x)$ for a.e. $x \in[0, \pi]$ and all $u \in \mathbf{R}$ and some $\alpha \in L^{1}[0, \pi]$. The same degree arguments will imply the existence of a solution for (5.8) if the set of possible solutions of the family of equations 


$$
\begin{gathered}
-\frac{d^{4} u}{d x^{4}}+[(1-\lambda)(\Gamma(x)+\eta)+\tilde{\gamma}(x, u(x))] u(x)=-\lambda h(x, u(x))+\lambda e_{1}(x), \lambda \in(0,1), \\
u^{\prime}(0)=u^{\prime}(\pi)=u^{\prime \prime \prime}(0)=u^{\prime \prime \prime}(\pi)=0,
\end{gathered}
$$

is, a priori, bounded in $C[0, \pi]$ independently of $\lambda \in(0,1)$. Let $u(x)$ be a solution of $(5.12)$ for some $\lambda \in(0,1)$. Since

$$
0 \leq(1-\lambda)(\Gamma(x)+\eta)+\lambda \tilde{\gamma}(x, u(x)) \leq \Gamma(x)+\eta
$$

for a.e. $x \in[0, \pi]$, with $\bar{\Gamma}+\eta<\frac{4}{\pi^{2}}$, and since

$$
\left|e_{1}-h(\cdot, u(\cdot))\right|_{L^{1}} \leq\left|e_{1}\right|_{L^{1}}+|\alpha|_{L^{1}},
$$

it follows from Lemma 4 that

$$
\begin{aligned}
{\left[1-\frac{\pi^{2}}{4}(\bar{\Gamma}+\eta)\right]\left|\frac{d^{4} u}{d x^{4}}\right|_{L^{1}}^{2} } & \leq 2\left(\left|e_{1}\right|_{L^{1}}+|\alpha|_{L^{1}}\right)\left|\frac{d^{4} u}{d x^{4}}\right|_{L^{1}} \\
& +(\bar{\Gamma}+\eta)\left(\left|e_{1}\right|_{L^{1}}+|\alpha|_{L^{1}}\right)|u|_{L^{-}}+3\left(\left|e_{1}\right|_{L^{1}}+|\alpha|_{L^{1}}\right)^{2} .
\end{aligned}
$$

Also, we see as in the proof of Theorem 2 that there exists a $\tau \in[0, \pi]$ such that

$$
r<u(\tau)<R .
$$

Next, since it is easy to obtain the solution $u$, with $\bar{u}=0$, of the linear problem $\frac{d^{4} u}{d x^{4}}=y$, $u^{\prime}(0)=u^{\prime}(\pi)=u^{\prime \prime \prime}(0)=u^{\prime \prime \prime}(\pi)=0$, for any given $y \in L^{1}[0, \pi]$ with $\vec{y}=0$, we see that there exist constants $\delta_{1}>0, \delta_{2}>0$ such that

$$
|\tilde{u}|_{L^{-}} \leq \delta_{1}\left|\frac{d^{4} u}{d x^{4}}\right|_{L^{1}}
$$

and

$$
\left|u^{\prime}\right|_{L^{-}} \leq \delta_{2}\left|\frac{d^{4} u}{d x^{4}}\right|_{L^{1}}
$$

Using (5.15) in (5.13), we find that

$$
\begin{aligned}
{\left[1-\frac{\pi^{2}}{4}(\bar{\Gamma}+\eta)\right]\left|\frac{d^{4} u}{d x^{4}}\right|_{L^{1}}^{2} \leq } & \left(\left|e_{1}\right|_{L^{1}}+|\alpha|_{L^{1}}\right)\left(2+\delta_{1}(\bar{\Gamma}+\eta)\right)\left|\frac{d^{4} u}{d x^{4}}\right|_{L^{1}} \\
& +(\bar{\Gamma}+\eta)\left(\left|e_{1}\right|_{L^{1}}+|\alpha|_{L^{1}}\right)|\bar{u}|+3\left(\left|e_{1}\right|_{L^{1}}+|\alpha|_{L^{1}}\right)^{2} .
\end{aligned}
$$

Also, it follows from (5.14),(5.16) that

$$
|u(x)|=\left|u(\tau)+\int_{\tau}^{x} u^{\prime}(s) d s\right| \leq \max (-r, R)+\pi\left|u^{\prime}\right|_{L^{-}} \leq \max (-r, R)+\pi \delta_{2}\left|\frac{d^{4} u}{d x^{4}}\right|_{L^{1}}
$$


so that

$$
|\bar{u}| \leq \max (-r, R)+\pi \delta_{2}\left|\frac{d^{4} u}{d x^{4}}\right|_{L^{1}} .
$$

Finally, it follow: from (5.15), (5.17), and (5.18) that there exists a constant $\rho$, independent of $\lambda \in(0,1)$ such that

$$
|u|_{L-} \leq \rho \text {. }
$$

Remark 3. If there is no $L^{\infty}$-resonance, (i.e., $\Gamma_{0}=\Gamma_{\infty}=0$ ), Theorem 3 improves the condition on $\bar{\Gamma}$ to $\bar{\Gamma}<\frac{4}{\pi^{2}}$, when compared to Theorem 2 , where $\bar{\Gamma}$ would be required to be such that $\bar{\Gamma}<\frac{3}{2 \pi^{2}}$.

Remark 4. If $p(x)$ in Lemma 4 satisfies in addition that for a given $\eta>0, p(x) \geq \eta>0$ for a.e. $x \in[0, \pi]$ and $\bar{\Gamma}<\frac{4}{\pi^{2}}$, then it follows easily from inequality (5.3) that the boundary value problem (5.1) has at most one solution.

We need the following theorem of Mawhin (Theorem 1, [2]) which we state here as a proposition.

PROPOSITION 1. Let $X$ and $Z$ be normed vector-spaces such that $C[0, \pi] \subset X \subset Z \subset L^{1}[0, \pi]$. Let $L: \operatorname{dom}(L) \subset X \rightarrow Z$ be a linear Fredholm operator of index zero such that $D(L) \subset C[0, \pi]$, $\operatorname{ker} L=\{u \in D(L) \mid u$ is constant on $D\}, \operatorname{Im} L=\left\{v \in Z \mid \int_{0}^{\pi} v(x) \mathrm{d} x=0\right\}$. Let $g:[0, \pi] \times \mathbf{R} \rightarrow \mathbf{R}$ be a function such that for a.e. $x \in[0, \pi], g(x, \cdot)$ is non-decreasing and that the corresponding Nemytskii operator $N: X \rightarrow Z$, defined by $(N u)(x)=g(x, u(x)), x \in[0, \pi]$ is L-compact. Further, suppose that the canonical injection $J: X \rightarrow Z$ is $L$-compact and $h \in Z$ be given.

Let, now, there exist a positive measurable function $a:[0, \pi] \rightarrow \mathbf{R}$ such that $\operatorname{ker}(L+A)=\{0\}$, where $A: X \rightarrow Z$ is defined by $A u(x)=a(x) u(x)$, and there exists a real number $R_{1}>0$ and $a \delta, 0 \leq \delta<1$ such that

$$
L u+(1-\lambda) A u+\lambda N u=\lambda h, \quad \lambda \in(0,1)
$$

implies

$$
|\tilde{u}|_{L^{-}} \leq R_{1}+\delta|\bar{u}|
$$

Then the equation

$$
L u+N u=h,
$$

has at least one solution if and only if $\bar{h} \in \operatorname{Im} \bar{g}$ where $\bar{g}: \mathbf{R} \rightarrow \mathbf{R}$ is defined by

$$
\bar{g}(v)=\frac{1}{\pi} \int_{0}^{\pi} g(x, v) \mathrm{d} x .
$$

THEOREM 4. Let $g:[0, \pi] \times \mathbf{R} \rightarrow \mathbf{R}$ be a function satisfying Caratheodory's conditions and suppose that for a.e. $x \in[0, \pi], g(x, u)$ is nondecreasing in $u$. Let $\Gamma \in L^{1}[0, \pi]$ be as in Theorem 2 or 3 and

$$
\lim _{|u| \rightarrow \infty} \sup ^{-1} g(x, u) \leq \Gamma(x)
$$

uniformly for a.e. $x \in[0, \pi]$.

Then, for $e \in L^{1}[0, \pi]$, given the boundary value problem

$$
\begin{gathered}
-\frac{d^{4} u}{d x^{4}}+g(x, u(x))=e(x), \\
u^{\prime}(0)=u^{\prime}(\pi)=u^{\prime \prime \prime}(0)=u^{\prime \prime \prime}(\pi)=0,
\end{gathered}
$$


has at least one solution if and only if

$$
\frac{1}{\pi} \int_{0}^{\pi} e(x) \mathrm{d} x \in \operatorname{Im}\left(\frac{1}{\pi} \int_{0}^{\pi} g(x, \cdot) \mathrm{d} x\right)
$$

In case $\Gamma$ satisfies the conditions of Theorem 2, Theorem 4 follows from Proposition 1 in view of (4.12) with

$$
L: \operatorname{dom} L \subset C[0, \pi] \rightarrow L^{1}[0, \pi]
$$

by

$$
\operatorname{dom} L=\left\{u \in W^{4,1}[0, \pi] \mid u^{\prime}(0)=u^{\prime}(\pi)=u^{\prime \prime \prime}(0)=u^{\prime \prime \prime}(\pi)=0\right\}
$$

and

$$
L u=-\frac{d^{4} u}{d x^{4}} \text { for } u \in \operatorname{dom} L
$$

and $A: C[0, \pi] \rightarrow L^{1}[0, \pi]$ defined by $(A u)(x)=(\Gamma(x)+\eta) u(x)$, where $\eta=\frac{1}{2}\left[\delta\left(\Gamma_{0}\right)-\right.$ $\left.\pi^{2} / 3\left|\Gamma_{1}\right|_{L^{1}}-\left|\Gamma_{\infty}\right|_{L^{-}}\right]$. We note that $\operatorname{ker}(L+A)=\{0\}$ by Corollary 1.

And in case $\Gamma$ satisfies the conditions of Theorem 3, Theorem 4 again follows from Proposition 1 in view of (5.15),(5.17) with $L$ and $A$ as in the above paragraph except now $\eta=\frac{1}{2}\left(\frac{4}{\pi^{2}}-\bar{\Gamma}\right)$ and Remark 4 implies $\operatorname{ker}(L+A)=\{0\}$ in this case.

Example. It is easy to see that the boundary value problem

$$
\begin{gathered}
-\frac{d^{4} u}{d x^{4}}+u(x)=\cos x, \quad 0<x<\pi, \\
u^{\prime}(0)=u^{\prime}(\pi)=u^{\prime \prime \prime}(0)=u^{\prime \prime \prime}(\pi)=0,
\end{gathered}
$$

has no solution, even though

$$
0=\frac{1}{\pi} \int_{0}^{\pi} \cos x \mathrm{~d} x \in \mathbf{R}=\operatorname{Im} \text { (identity) }
$$

(Note here $g(x, u)=u$ so that $\frac{1}{\pi} \int_{0}^{\pi} g(x, u) \mathrm{d} x=u$ for $u \in \mathbf{R}$.)

This example points out the necessity of some conditions on $\Gamma$ in Theorem 4.

\section{ACKNOWLEDGMENTS}

The author thanks Dr. M. K. Kwong for his helpful observation about the validity of Wirtinger's inequality (2.11). This work was supported in part by the Applied Mathematical Sciences subprogram of the Office of Energy Research, U.S. Department of Energy, under Contract W-31-109-Eng-38 under the Faculty Research Leave Program at Argonne National Laboratory. The author's permanent address is: Department of Mathematical Sciences, Northern Illinois University, DeKalb, IL 60115. 


\section{REFERENCES}

1. GUPTA, C. P., and MAWHIN, J., Asymptotic Conditions at the First Two Eigenvalues for the Periodic Solutions of Liénard Differential Equations and an Inequality of E. Schmidt, Zeit. für Analysis und ihre Anwendungen, 3 (1984), pp. 33-42.

2. MAWHIN, J. Le role de la regularite dans les problemes nonlinearires resonants, in Publications de l'U.E.R. de Mathematiques pures et appliquées, Université de Lille, fascimile 2, Vol. 5, 1983.

3. MAWHIN, J., and WARD, J. R., Nonuniform Non-Resonance Conditions at the Two First Eigenvalues for Periodic Solutions of Forced Lienard and Duffing Equations, Rocky Mountain J. Math., 12 (1982), pp. 643-652.

4. MAWHIN, J., and WARD, J. R., Periodic Solutions of Some Forced Lienard Differential Equations at Resonance, Arch. Math. (Basel), 41 (1983), pp. 337-351.

5. ROUCHE, N., and MAWHIN. J., Ordinary Differential Equation Stability and Periodic Solutions, Pitman, Boston, 1980.

6. GAINES, R. E., and MAWHIN, J., Coincidence Degree and Nonlinear Differential Equations, Lecture Notes in Mathematics, No. 568, Springer-Verlag, Berlin, 1977.

7. MAWHIN, J., Topological Degree Methods in Nonlinear Boundary Value Problems CBMS-Regional Conference Series in Math., American Mathematical Society, Providence, Rhode Island.

8. MAWHIN, J., Landesman-Lazer Type Problems for Nonlinear Equations, Conf. Sem. Univ. Bari., no. 147 (1977).

9. MAWHIN, J., Compacité Monotonie, et Convexite'dans l'etude de Problémes aux Limites SemiLineaires, Semi Anal. Moderne, No. 19 (1981), Université de Sherbrooke, Québec, Canada. 


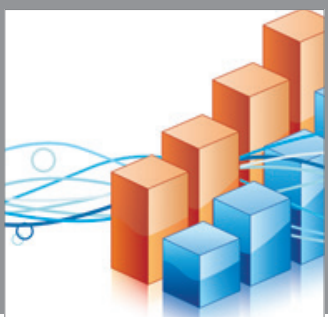

Advances in

Operations Research

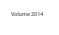

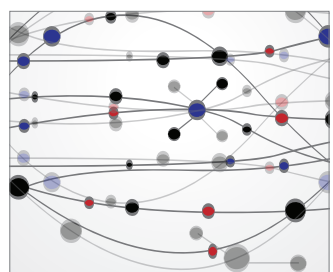

\section{The Scientific} World Journal
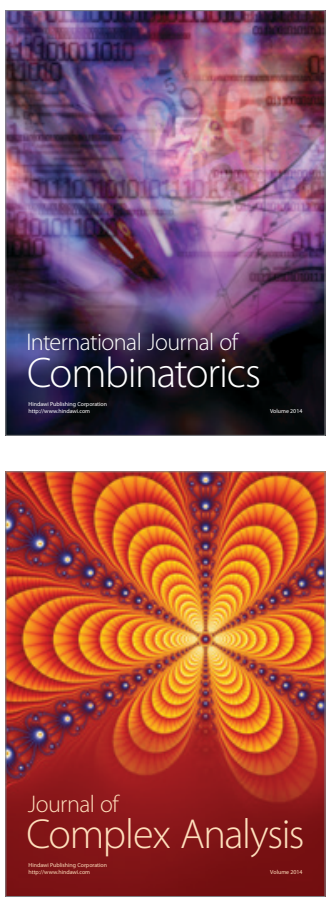

International Journal of

Mathematics and

Mathematical

Sciences
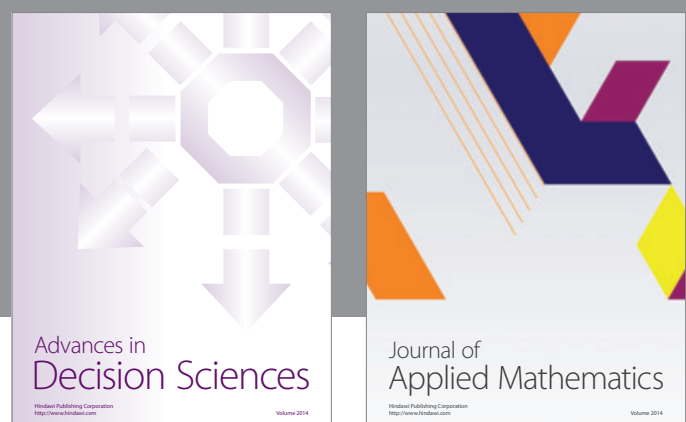

Journal of

Applied Mathematics
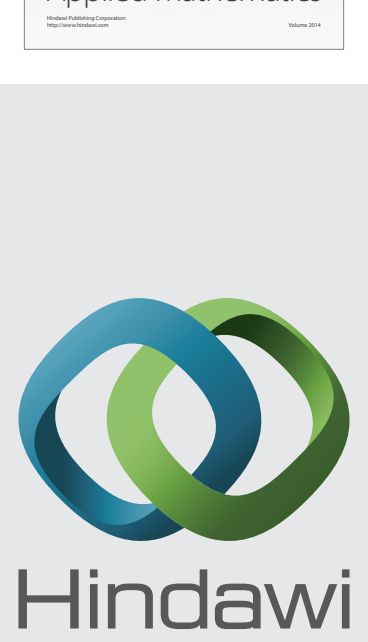

Submit your manuscripts at http://www.hindawi.com
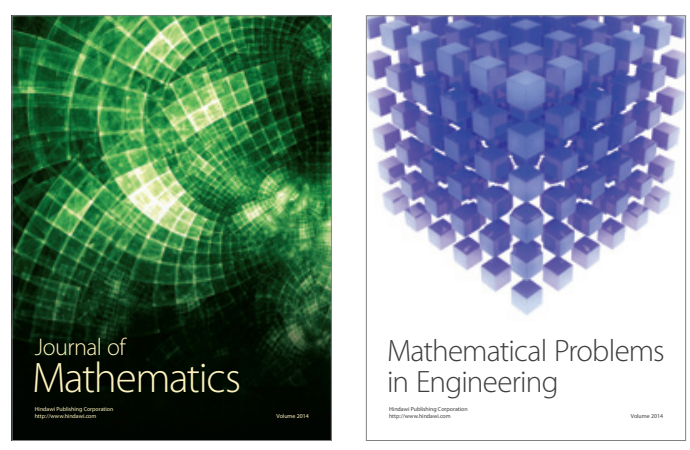

Mathematical Problems in Engineering
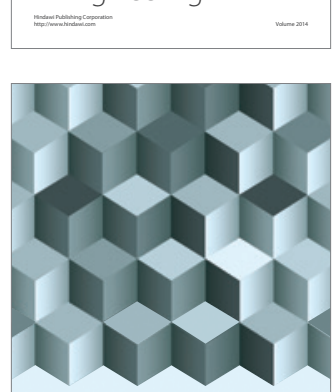

Journal of

Function Spaces
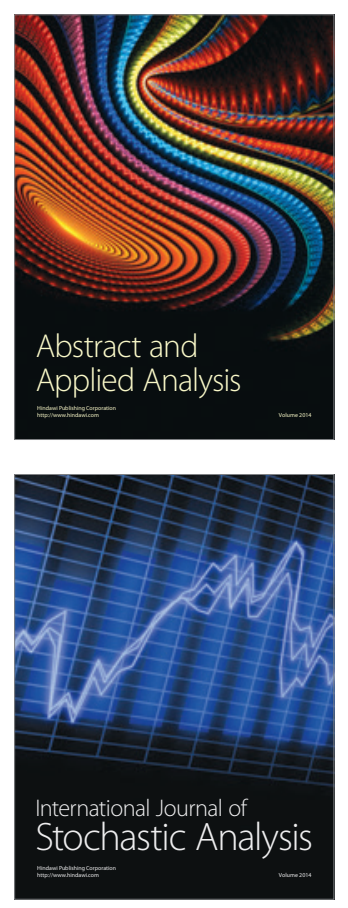

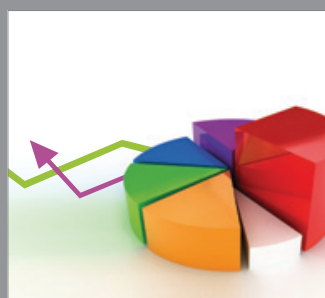

ournal of

Probability and Statistics

Promensencen
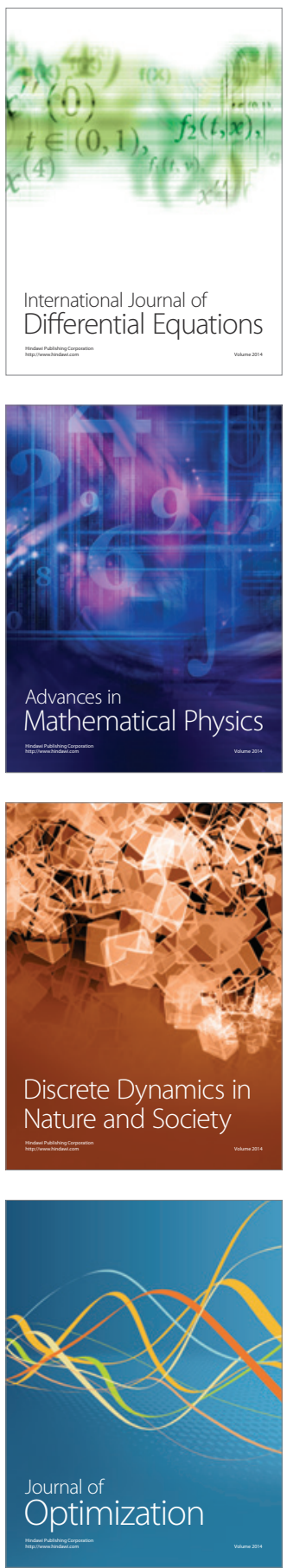